\section{Tendências na incidência e mortalidade por acidentes de trabalho no Brasil, 1998 a 2008}

\author{
Trends in incidence and mortality due to \\ occupational accidents in Brazil, 1998-2008
}

\author{
Tendencias en la incidencia y la mortalidad por \\ accidentes de trabajo en Brasil, 1998-2008
}

\section{Abstract}

The objective was to evaluate trends in incidence and mortality due to occupational accidents in Brazil from 1998 to 2008. This was a time-trend series study that included cases of occupational accidents recorded in official Federal government statistics. The authors calculated annual percentage changes (APC) in incidence and mortality rates with the Joinpoint method using the calendar year as a regressor variable. There was a significant downward trend in incidence rates of occupational accidents, and the same trend was observed in typical occupational accidents. However, the number of cases increased during this period. There was a statistically significant upward trend in the incidence and number of cases of commuting accidents. The number of deaths and mortality rates showed a downward trend. Several factors may have contributed to the decline in incidence and mortality rates for occupational accidents, including improvement in working conditions, a shift in the economy from industry to services, underreporting of occupational accidents, and outsourcing of services. The increase in commuting accidents suggests the influence of violence in urban areas.

Occupational Accidents; Occupational Mortality; Time Series Studies
Flávia Souza e Silva de Almeida 1

Luiz Carlos Morrone ${ }^{\dagger}$

Karina Braga Ribeiro ${ }^{1}$

\section{Resumo}

O objetivo do estudo foi avaliar as tendências na incidência e mortalidade por acidentes de trabalho no Brasil, de 1998 a 2008. Trata-se de estudo ecológico de séries temporais, que incluiu, para a análise, os acidentes de trabalho registrados nas estatísticas oficiais do Governo Federal. Foram calculadas as variações percentuais anuais (APC) nas taxas de incidência e de mortalidade, através da modelagem pelo método Joinpoint, usando o ano calendário como variável regressora. Observouse tendência significativa de decréscimo na taxa de incidência de acidentes de trabalho, o mesmo ocorrendo para os acidentes de trabalho típicos. Para ambos, o número de casos aumentou nesse período. O número de casos de acidentes de trabalho de trajeto e sua incidência mostraram tendência significativa de aumento. O número de óbitos e a taxa de mortalidade registraram tendência de decréscimo. São discutidos como fatores contribuintes para o declínio das taxas de incidência de acidentes de trabalho e de mortalidade por esta causa: melhoria das condições de trabalho, maior crescimento do setor de serviços do que do setor industrial, subnotificação dos acidentes de trabalho e terceirização dos serviços. O aumento dos acidentes de trabalho de trajeto sugere a influência da violência dos centros urbanos.

Acidentes de Trabalho; Mortalidade Ocupacional; Estudos de Séries Temporais 


\section{Introdução}

No Brasil, a saúde do trabalhador é atribuição do Sistema Único de Saúde (SUS), que compartilha as responsabilidades com os empregadores e trabalhadores (por meio de suas representações). Pelo Estado, atuam na saúde do trabalhador os Ministérios do Trabalho, da Previdência Social, da Saúde e do Meio Ambiente; a Justiça do Trabalho e a Promotoria Pública 1 .

Os acidentes de trabalho representam um problema de saúde pública em todo o mundo, principalmente por envolverem pessoas jovens e em idade produtiva, podendo gerar ocorrências incapacitantes e até mesmo fatais, com importantes repercussões sociais e econômicas 2,3. Mesmo em países com importantes avanços no campo da prevenção de acidentes de trabalho, os custos são raramente contabilizados. Estimase que $4 \%$ do Produto Interno Bruto (PIB) sejam perdidos por doenças e agravos ocupacionais, podendo aumentar para $10 \%$ nos países em desenvolvimento. Nos Estados Unidos, em 1992, foi estimado um custo total de US\$ 145,37 bilhões decorrente de acidentes fatais e não fatais 4 . No Anuário Estatístico da Previdência Social de 2008 , há relato de que $2,8 \%$ de todas as despesas do Instituto Nacional do Seguro Social (INSS) devem-se aos benefícios relacionados a acidentes de trabalho, perfazendo um montante de $\mathrm{R} \$ 6,3$ bilhões.

A incidência de acidentes de trabalho no Brasil tem diminuído de forma significativa nas últimas décadas. No entanto continua alta em comparação com outros países, expressando a manutenção da precariedade das condições laborais e a baixa efetividade da regulamentação dos ambientes de trabalho ${ }^{6}$. As diferenças entre os países não se devem apenas a aspectos individuais dos trabalhadores; também estão relacionadas às políticas de proteção do trabalhador, tanto na sua formulação e organização, quanto na efetivação de suas variadas dimensões: técnicas, econômicas, sociais, culturais e políticas 7 .

Segundo Wünsch Filho 8, a tendência de declínio da incidência de acidentes de trabalho entre 1970 e 1995 diz respeito tanto ao número absoluto (número de acidentes de trabalho que independe do número de segurados) quanto à incidência (relação entre o número de acidentes e o número de segurados, variando de acordo com este último). Em 1970, ocorriam 167 acidentes em cada grupo de mil trabalhadores segurados pela Previdência Social; em 1980, esta relação reduz-se a 78 por mil; em 1990, a 36 por mil; em 1994, atingiu 16 por mil.

No período entre 1970 e 1994, também houve diminuição do coeficiente de mortalidade anual por acidentes de trabalho, que passou de 31 para 14 por 100 mil trabalhadores 8 . Valores semelhantes foram apresentados por Machado \& Gomez ${ }^{9}$, com uma queda de 30,64 em 1970 para 19,84 por 100 mil trabalhadores em 1991.

A falta de notificação por parte das empresas é decorrente de vários fatores, entre os quais as frequentes mudanças de legislação, que tem sido o principal argumento para explicar tal fato $8,10,11,12$. Entretanto, outros fatores podem estar influenciando essa tendência, tais como a definição, identificação e o registro de acidentes de trabalho 7 . É escassa, na literatura científica brasileira, a discussão do efeito de medidas preventivas e educativas sobre a ocorrência de acidentes de trabalho, mas também é pouco provável que a acentuada redução observada seja somente a consequência de respostas a ações de prevenção executadas pelas empresas ou agências governamentais responsáveis 8,11 .

Considerando a magnitude da questão da ocorrência e do registro de acidentes de trabalho, que sofre interferências de diversas instâncias, como o progresso científico e tecnológico, assim como as transformações socioeconômicas, além da escassa literatura recente sobre os acidentes de trabalho no Brasil, procuramos descrever as tendências na incidência e mortalidade por acidentes de trabalho no Brasil, no período de 1998 a 2008.

\section{Métodos}

Este é um estudo ecológico de séries temporais que incluiu, para a análise da incidência, os casos de acidentes de trabalho ocorridos entre 1998 e 2008 e, para o estudo da mortalidade, os óbitos por esta causa registrados no mesmo período. A base de dados foi levantada a partir da consulta ao Departamento de Informática do SUS (DATASUS), que utiliza a fonte do Ministério da Previdência Social (MPS). Por ter sido utilizada base de dados secundários, justifica-se a ausência de encaminhamento do estudo ao Comitê de Ética em Pesquisa.

Foram utilizadas as informações dos trabalhadores segurados pelo INSS. O denominador correspondente ao número médio de contribuintes empregados/ano; não houve a inclusão de dados de segurados especiais. Para o numerador, foi considerado o número total de acidentes, assim como os óbitos por acidentes de trabalho; não foram utilizados os casos sem Comunicação de Acidente de Trabalho (CAT), determinado pelo Nexo Técnico Epidemiológico Previdenciário (NTEP), em face da pequena série temporal deste. As doenças relacionadas ao trabalho, por 
apresentarem diferente caracterização, não foram consideradas neste estudo.

Consideramos as seguintes definições:

- Acidentes típicos: são os acidentes decorrentes da característica da atividade profissional desempenhada pelo segurado acidentado;

- Acidentes de trajeto: são os acidentes ocorridos no trajeto entre a residência e o local de trabalho do segurado e vice-versa;

- Óbitos por acidente de trabalho: número total de óbitos decorrentes dos acidentes do trabalho.

As taxas de incidência foram ajustadas por faixa etária, considerando a população padrão mundial proposta por Segi 13. O ajuste da taxa de mortalidade permite eliminar (ou minimizar) o efeito de diferenças etárias entre populações (ou na mesma população em períodos distintos), a fim de que diferenças geográficas ou temporais não possam ser atribuídas a diferenças na estrutura etária.

Foram calculadas as variações percentuais anuais (APC) nas taxas de incidência e mortalidade, através da modelagem pelo método Jointpoint, usando o ano calendário como variável regressora. As análises estatísticas foram realizadas com o software Jointpoint Regression Program, versão 3.3.1 (Statistical Research and Applications Branch, National Cancer Institute, Bethesda, Estados Unidos. http://srab.cancer. gov/joinpoint).

O modelo de regressão Joinpoint é um método que permite analisar tendências temporais (taxas de incidência, mortalidade, sobrevida ou prevalência), avaliando se, em alguns pontos (joinpoints), existem alterações do padrão de tendência observado. Assim como a regressão linear (que utiliza os mínimos quadrados), o Joinpoint (que utiliza como variável dependente uma transformação logarítmica da taxa) é usado para encontrar o melhor modelo; ele testa se vários segmentos de reta (com vários joinpoints) explicam melhor uma tendência no tempo do que uma reta única. O Joinpoint utiliza testes de permutação de Monte Carlo para comparar os diversos modelos (com $0,1,2,3$, 4 ou 5 joinpoints - a depender do tamanho da série temporal) e avaliar qual é o melhor 14.

Uma vez que o modelo é definido, a APC, para cada segmento, é calculada e usada não só para descrever e quantificar a tendência, mas também para avaliar se esta tendência é estatisticamente significativa. Neste caso, a hipótese nula é APC = 0 , isto é, as taxas não estão nem aumentando, nem diminuindo. Por isso, são apresentados os intervalos de 95\% de confiança (IC95\%).

Neste estudo, foram incluídos somente os trabalhadores segurados acima de 25 anos de idade, para evitar uma diminuição na série temporal, pois, abaixo desta faixa, etária houve mudanças na forma de sua apresentação.

Para controlar possível efeito da flutuação da população de segurados, analisamos também a tendência do número absoluto de casos de acidentes de trabalho e de óbitos.

\section{Resultados}

No período de 1998 a 2008, foram registrados, no Brasil, 3.475.504 acidentes de trabalho, incluídos os acidentes de trabalho típicos e os de trajeto, em trabalhadores segurados acima de 25 anos. A maior taxa de incidência ajustada (18,3 acidentes por mil trabalhadores segurados) foi observada no ano 2000 , enquanto a menor (14,6 por mil) foi registrada em 2008 (Tabela 1 ).

Observou-se tendência significativa de decréscimo na incidência de acidentes de trabalho no período estudado, considerando ambos os sexos, com APC de -1,6 (IC95\%: -2,5; -0,7) (Tabela 2). No período de 1998 a 2001, porém, observouse tendência de declínio no número dos casos de acidentes de trabalho sem significância estatística (APC = -1,6; IC95\%: -7,9; 5,2) e aumento estatisticamente significativo no período de 2001 a 2008 (APC = 6,9; IC95\%: 5,3; 8,6). Considerando todo o período, o número de casos de acidentes de trabalho em ambos os sexos teve tendência de aumento significativo, com variação percentual anual média (AAPC) de 4,3 (IC95\%: 2,4; 6,2) (Tabela 3).

Em relação aos acidentes de trabalho típicos, foram registradas 3.007.826 ocorrências no Brasil, ao longo do período investigado. A maior taxa de incidência ajustada (16,2 acidentes por mil trabalhadores segurados) foi observada no ano 2000 , enquanto a menor (12,2 por mil) foi registrada em 2008 (Tabela 1 ).

A mesma tendência verificada para acidentes de trabalho foi registrada para os acidentes de trabalho típicos, isto é, declínio na tendência da taxa de incidência (APC = -2,3; IC95\%: $-3,3 ;-1,3$ ) (Tabela 2) e aumento da tendência do número de casos considerando todo o período (AAPC = 3,0; IC95\%: 0,2; 5,8) (Tabela 3). Contudo, para o número de casos de acidentes de trabalho típicos, observou-se declínio não significativo no período de 1998 a 2001 (APC = -3,9; IC95\%: -11,4; $4,2)$, seguido de aumento significativo no período de 2001 a 2005 (APC = 8,4; IC95\%: 0,5; 17,0) e aumento não significativo no período de 2005 a 2008 (APC = 3,1; IC95\%: -3,8; 10,4).

Ainda no período estudado, foram registrados 467.678 acidentes de trabalho de trajeto no Brasil. A maior taxa de incidência ajustada (2,5 
Tabela 1

Número de casos, óbitos e taxas de incidência e mortalidade de acidentes de trabalho entre trabalhadores segurados de ambos os sexos. Brasil, $1998-2008$.

\begin{tabular}{|c|c|c|c|c|c|c|c|c|}
\hline \multirow[t]{2}{*}{ Ano } & \multicolumn{2}{|c|}{$\begin{array}{l}\text { Acidentes de trabalho } \\
\text { (típico + trajeto) }\end{array}$} & \multicolumn{2}{|c|}{$\begin{array}{c}\text { Acidentes de trabalho } \\
\text { (típico) }\end{array}$} & \multicolumn{2}{|c|}{$\begin{array}{c}\text { Acidentes de trabalho } \\
\text { (trajeto) }\end{array}$} & \multicolumn{2}{|c|}{ Mortalidade } \\
\hline & Casos & $\begin{array}{c}\text { Taxa de } \\
\text { incidência * }\end{array}$ & Casos & $\begin{array}{c}\text { Taxa de } \\
\text { incidência * }\end{array}$ & Casos & $\begin{array}{c}\text { Taxa de } \\
\text { incidência * }\end{array}$ & Óbitos & $\begin{array}{c}\text { Taxa de } \\
\text { mortalidade * }\end{array}$ \\
\hline 1998 & 270.657 & 17,6 & 244.631 & 15,9 & 26.026 & 1,7 & 2.700 & 21,2 \\
\hline 1999 & 275.172 & 17,3 & 246.668 & 15,5 & 28.504 & 1,8 & 3.244 & 22,5 \\
\hline 2000 & 261.897 & 18,3 & 231.846 & 16,2 & 30.051 & 2,2 & 2.655 & 20,6 \\
\hline 2001 & 245.738 & 15,4 & 215.996 & 13,5 & 29.742 & 1,9 & 2.369 & 17,7 \\
\hline 2002 & 283.365 & 16,8 & 247.726 & 14,7 & 35.639 & 2,2 & 2.528 & 17,4 \\
\hline 2003 & 288.946 & 15,3 & 251.042 & 13,3 & 37.904 & 2,0 & 2.257 & 13,4 \\
\hline 2004 & 334.026 & 16,4 & 288.082 & 14,1 & 45.944 & 2,3 & 2.419 & 14,4 \\
\hline 2005 & 358.283 & 16,4 & 306.995 & 14,0 & 51.288 & 2,4 & 2.313 & 12,2 \\
\hline 2006 & 371.749 & 16,5 & 315.132 & 14,0 & 56.617 & 2,5 & 2.325 & 12,3 \\
\hline 2007 & 380.962 & 15,2 & 321.216 & 12,8 & 59.746 & 2,4 & 2.310 & 10,2 \\
\hline 2008 & 404.709 & 14,6 & 338.492 & 12,2 & 66.217 & 2,4 & 2.309 & 9,9 \\
\hline
\end{tabular}

* Ajustada por idade.

Fonte: Ministério da Previdência e Assistência Social (MPAS) e Coordenação Geral de Estatística e Atuária (CGEA/DATAPREV).

Tabela 2

Variação percentual anual (APC) nas taxas de incidência e mortalidade de acidentes de trabalho entre trabalhadores segurados de ambos os sexos. Brasil, 1998-2008.

\begin{tabular}{|c|c|c|c|}
\hline Tipo & APC & IC95\% & Período \\
\hline Taxa de incidência de acidentes de trabalho (típico + trajeto) & $-1,6$ & $-2,5 ;-0,7$ & $1998-2008$ \\
\hline Taxa de incidência de acidentes de trabalho (típico) & $-2,3$ & $-3,3 ;-1,3$ & $1998-2008$ \\
\hline Taxa de incidência de acidentes de trabalho (trajeto) & 3,2 & 1,$7 ; 4,8$ & $1998-2008$ \\
\hline Taxa de mortalidade por acidente de trabalho & $-8,3$ & $-9,6 ;-7,0$ & $1998-2008$ \\
\hline
\end{tabular}

IC95\%: intervalo de 95\% de confiança.

Tabela 3

Variação percentual anual (APC) ou média da variação percentual anual (AAPC) dos números de casos e óbitos relacionados a acidentes de trabalho entre trabalhadores segurados de ambos os sexos. Brasil, 1998-2008.

\begin{tabular}{lccc}
\hline Tipo & APC/AAPC & IC95\% & Período \\
\hline Casos de acidentes de trabalho (típico + trajeto) & 4,3 & 2,$4 ; 6,2$ & $1998-2008$ \\
Casos de acidentes de trabalho (típico) & 3,0 & 0,$2 ; 5,8$ & $1998-2008$ \\
Casos de acidentes de trabalho (trajeto) & 10,5 & 9,$3 ; 11,7$ & $1998-2008$ \\
Obitos por acidente de trabalho & $-2,5$ & $-4,2 ;-0,8$ & $1998-2008$ \\
\hline
\end{tabular}

IC95\%: intervalo de 95\% de confiança. 
acidentes por mil trabalhadores segurados) foi registrada em 2006, enquanto a menor (1,7 por mil) foi observada em 1998 (Tabela 1).

Observou-se tendência significativa de aumento nas taxas de incidência (APC = 3,2; IC95\%: $1,7 ; 4,8)$ (Tabela 2) e no número absoluto de casos $(\mathrm{APC}=10,5$; IC95\%: 9,3; 11,7) (Tabela 3$)$.

Em relação aos óbitos por acidentes de trabalho, foram registrados 27.429, no Brasil, de 1998 a 2008. A maior taxa de mortalidade ajustada $(22,5$ óbitos por 100 mil trabalhadores segurados) foi observada no ano 1999, enquanto a menor (9,9 por 100 mil) foi registrada em 2008 (Tabela 1).

Para a mortalidade por acidentes de trabalho, registrou-se tendência de decréscimo, tanto para a taxa $($ APC $=-8,3$; IC95\%: $-9,6 ;-7,0)$ (Tabela 2 ), quanto para o número de óbitos ( $\mathrm{APC}=-2,5$; IC95\%: -4,2; -0,8) (Tabela 3).

\section{Discussão}

Este estudo observou tendência significativa de decréscimo na incidência de acidentes de trabalho para ambos os sexos, achado similar aos de outros estudos, que demonstraram declínio no período de 1970 a $1995^{8}$ e de 1996 a 2000 6. Os fatores identificados como contribuintes para essa tendência foram a perda da contribuição do setor secundário para o PIB do Brasil (Instituto Brasileiro de Geografia e Estatística. Séries Estatísticas. http://seriesestatisticas.ibge.gov.br, acessado em 02/Mai/2011), além da flexibilização e da desregulamentação das formas de contratação do empregado, como a terceirização.

A terceirização no Brasil, iniciada com a vinda das empresas multinacionais, visa à diminuição dos custos administrativos de pessoal, para que a empresa se dedique somente à sua atividade fim e repasse os custos para a empresa subcontratada. Com o deslocamento da força de trabalho para as subcontratadas há falta de controle sobre as práticas de terceirização e pode haver aumento dos acidentes de trabalho. No entanto, pela distância entre empregador e empregado, esses acidentes podem não ser registrados 15 . Também pode ter ocorrido um maior investimento em segurança e saúde do trabalhador, mas existem poucos estudos que avaliam os programas de saúde ocupacional. Estes, além de raros, enfocam desfechos ou programas específicos de intervenção sobre agentes de risco ou enfermidades e agravos isolados, apresentando, em geral, problemas metodológicos 16 .

Ressalta-se que, apesar de a taxa de incidência de acidentes de trabalho ter declinado, o número de casos aumentou no Brasil nesse período. Foi observado, ainda, aumento do número de segurados pelo INSS, justificado pelo aumento da formalidade do trabalho observado a partir de 2002 17,18. Embora tenha sido verificada redução na incidência, a elevação, no período, do número de acidentes de trabalho indica que não deve ter ocorrido efetiva redução de riscos nas empresas decorrente da melhora das condições de trabalho.

Para os acidentes de trabalho típicos, observou-se a mesma tendência da taxa de incidência de todos os acidentes de trabalho, pois esse tipo de acidente é o que contribui para o padrão da tendência de acidentes laborais no Brasil. Esta mesma tendência foi demonstrada por Binder et al. 10, no período de 1995 a 1999, para o Município de Botucatu (São Paulo); todavia, neste mesmo período, o número de casos de acidentes de trabalho típicos aumentou, mostrando que ainda é relevante para o adoecimento dos trabalhadores.

O aumento observado na incidência de acidentes de trabalho de trajeto no Brasil, para ambos os sexos, já foi apresentado por Binder et al. ${ }^{10}$ para o Município de Botucatu, entre os anos de 1997 e 1998. Embora tenha voltado a diminuir em 1999, esse resultado indica que a violência e o crescimento urbano passaram a atingir os trabalhadores, principalmente nos grandes centros. Em Campinas (São Paulo), verificou-se que a violência urbana ganha relevância como fator desencadeante para os acidentes de trabalho, principalmente no que se refere aos acidentes de trajeto, com a percepção de que tal quadro seja encontrado em todas as grandes cidades brasileiras 19 .

Entre os fatores que podem ter contribuído para o crescimento do número de acidentes de trajeto no Brasil está o aumento do número de veículos por 100 habitantes, que passou de 17,4 em 2000 para 28,5 em 2008. Este achado também é corroborado pelo aumento do número de vitimas de morte em acidentes de transportes terrestres: de 11,8 para 17,8 por 100 mil habitantes no mesmo período (Departamento Nacional de Trânsito. Registro Nacional de Acidentes e Estatísticas de Trânsito, 2008. http:/ /www.denatran.gov. br/frota.htm, acessado em 22/Fev/2012). Além disso, pode-se destacar a introdução da motocicleta como meio de transporte para o trabalho por sua rapidez e economia, refletindo no aumento do número de acidentes de moto de 1980 a 2003 e, especialmente, a partir de 1995 20,21. Houve, também, a partir da década de 1990, benefícios fiscais concedidos pelo Governo Federal para aquisição de motocicletas, a fim de gerar empregos e permitir que os jovens de baixa renda tivessem acesso a um meio de transporte motorizado. Entretanto, o ambiente de circulação não 
foi adequadamente preparado, de modo que as más condições das vias quanto à conservação, sinalização, velocidade média do fluxo, drenagem e iluminação também aumentam o risco de acidentes envolvendo motociclistas 21 .

Apesar de termos observado decréscimo na taxa de mortalidade por acidentes de trabalho no Brasil, ela ainda se mantém elevada em relação à de outros países 9 . Em 2001, a taxa de mortalidade registrada no Brasil era de 17,7, enquanto, na Coreia do Sul, era de 12,8 e, nos Estados Unidos, de 3,5 22; em 2002, na Espanha, a taxa era de 6,1 23 (todos os coeficientes são expressos por $100 \mathrm{mil}$ trabalhadores).

A variação percentual anual nas taxas de mortalidade por acidente de trabalho no Brasil foi de $-8,28 \%$ no período de 1998 a 2008 . Outros países apresentaram declínio menor, tais como: Itália (-3,4\% ao ano; 1951-1998) 24, Espanha $(-4,3 \%$ ao ano; $1992-2002) 23$, Coreia do Sul $(-5 \%$ ao ano; 1998-2001) 22 e Estados Unidos (-2\% ao ano; 1998-2001) 22. O menor declínio nesses países deve-se, possivelmente, ao fato de já terem menores taxas de mortalidade por acidente de trabalho, ou seja, encontram-se praticamente já estabilizadas.

A redução na mortalidade pode ser explicada pela mudança da economia, que transferiu a mão de obra do setor secundário para o setor terciário, o que pode ter levado a uma menor exposição aos riscos ocupacionais e, consequentemente, a um menor número de óbitos 18 . Por outro lado, o processo de terceirização pode ter determinado o deslocamento das atividades mais perigosas para este setor. Segundo Marcelino 15, as empresas terceiras que assumiram estas atividades poderiam estar trabalhando com mão de obra não registrada, eliminando o registro de acidentes fatais, que podem estar sendo transferidos para os setores informais da economia. No entanto, como já referido, não se observa aumento na informalidade do trabalho desde 2002 17,18. Além disso, o trabalho informal não contribui para o aumento do número de acidentes de trabalho, pois não há a CAT; sendo assim, esses trabalhadores não fazem parte da população estudada, já que somente $5 \%$ deles contribuem com a Previdência Social.

Uma das limitações deste estudo é o fato de ser baseado exclusivamente nos dados da Previdência Social, restringindo as conclusões possíveis a apenas uma parte da população trabalhadora, isto é, aos trabalhadores segurados, que contribuem com o INSS. Exclui, dessa forma, amplos segmentos da sociedade, tais como grande parte dos servidores públicos e a totalidade dos trabalhadores informais. Outra limitação é a definição da população em risco. O INSS considera como população total de trabalhadores segurados o número médio de contribuintes no ano, sendo possível haver trabalhadores que contribuíram somente alguns meses no ano.

Por fim, nossos achados mostram a necessidade de políticas públicas direcionadas à saúde do trabalhador, pois, apesar de as taxas de incidência e mortalidade por acidentes de trabalho estarem em declínio, o número de casos ainda é elevado. É preciso, também, reafirmar a importância do aperfeiçoamento do registro de acidentes de trabalho, independentemente da forma do vínculo empregatício. Devem ser promovidas ações para a redução do número de casos, como investimentos em segurança e saúde dos trabalhadores. 


\section{Resumen}

El objetivo fue evaluar las tendencias en la incidencia y la mortalidad por accidentes de trabajo en Brasil, de 1998 a 2008. Se trata de un estudio ecológico de series temporales que incluyó el análisis de los accidentes de trabajo registrados en las estadísticas oficiales del Gobierno Federal. Se calculó el cambio porcentual anual en las tasas de incidencia y en las tasas de mortalidad por el método Joinpoint, utilizando el año natural como variable regresora. Hubo una tendencia significativa de disminución de las tasas de incidencia por accidentes de trabajo, lo mismo sucedió con los accidentes de trabajo típicos. Sin embargo, el número de casos ha aumentado. El número de casos de accidentes de trabajo de desplazamiento y sus tasas de incidencia mostró una tendencia en aumento. El número de muertes y tasas de mortalidad mostró una tendencia decreciente. Se discuten los factores que contribuyen a la disminución de tasas de incidencia y tasas de mortalidad por accidentes de trabajo: la mejora de las condiciones de trabajo, un mayor crecimiento del sector de servicios, en relación con el sector industrial, el subregistro de accidentes de trabajo, y servicios de outsourcing. El aumento de accidentes de trabajo durante el desplazamiento sugiere la influencia de la violencia en los centros urbanos.

Accidentes de Trabajo; Mortalidad Laboral; Estudios de Series Temporales

\section{Colaboradores}

F. S. S. Almeida contribuiu substancialmente para a concepção, design e redação do artigo, aquisição, análise e interpretação dos dados, revisão crítica do conteúdo intelectual, além da elaboração e aprovação final da versão a ser publicada. L. C. Morrone contribuiu substancialmente para a concepção do artigo, a análise e interpretação dos dados, revisão crítica do conteúdo intelectual e aprovação final da versão a ser publicada. K. B. Ribeiro contribuiu substancialmente para a aquisição, análise e interpretação dos dados, revisão crítica do conteúdo intelectual e aprovação final da versão a ser publicada.

\section{Referências}

1. Silva ZP, Barreto Júnior IF, Sant'ana MC. Saúde do trabalhador no âmbito municipal. São Paulo Perspect 2003; 17:47-57.

2. Santana VS, Maia AP, Carvalho C, Luz G. Acidentes de trabalho não fatais: diferenças de gênero e tipo de contrato de trabalho. Cad Saúde Pública 2003; 19:481-93.

3. Santana VS, Araújo Filho JB, Oliveira PRA, Branco AB. Acidentes de trabalho: custos previdenciários e dias de trabalho perdidos. Rev Saúde Pública 2006; 40:1004-12.

4. Leigh JP, Markowitz SB, Fahs M, Shin C, Landrigan PJ. Occupational injury and ilness in the United States: estimates of costs, morbidity and mortality. Arch Intern Med 1997; 157:1557-68.

5. Empresa de Tecnologia e Informações da Previdência Social. Anuário estatístico da previdência social: 2008. http://www.previdenciasocial.gov.br/ conteudoDinamico.php?id=850 (acessado em 02/ Mai/2011).

6. Santana VS, Nobre L, Waldvogel BC. Acidentes de trabalho no Brasil entre 1994 e 2004: uma revisão. Ciênc Saúde Coletiva 2005; 10:841-55.
7. Santana VS, Araújo-Filho JB, Silva M, AlbuquerqueOliveira PR, Barbosa-Branco A, Nobre LCC. Mortalidade, anos potenciais de vida perdidos e incidência de acidentes de trabalho na Bahia, Brasil. Cad Saúde Pública 2007; 23:2643-52.

8. Wünsch Filho V. Reestruturação produtiva e acidentes de trabalho no Brasil: estrutura e tendências. Cad Saúde Pública 1999; 15:41-52.

9. Machado JMH, Gomez CM. Acidentes de trabalho: uma expressão da violência social. Cad Saúde Pública 1994; 10 Suppl 1:74-87.

10. Binder MCP, Wludarski SL, Almeida IM. Estudo da evolução dos acidentes do trabalho registrados pela Previdência Social no período de 1995 a 1999, em Botucatu, São Paulo. Cad Saúde Pública 2001; 17:915-24.

11. Cordeiro R, Prestes SCC, Clemente APG, Diniz CS, Sakate M, Donalisio MR. Incidência de acidentes do trabalho não-fatais em localidade do Sudeste do Brasil. Cad Saúde Pública 2006; 22:387-93. 
12. Lucca SR, Mendes R. Epidemiologia dos acidentes do trabalho fatais em área metropolitana da região sudeste do Brasil, 1979-1989. Rev Saúde Pública 1993; 27:168-76.

13. Segi M. Cancer mortality for selected sites in 24 countries (1950-1957). Sendai: Tohoku University School of Medicine.

14. Kim HJ, Fay MP, Feuer EJ, Midthune DN. Permutation tests for joinpoint regression with application to cancer rates. Stat Med 2000; 19:335-51.

15. Marcelino PRP. Terceirização do trabalho no Brasil e na França. In: Anais do II Simpósio Estadual Lutas Sociais na América Latina. http://www.uel.br/grupo -pesquisa/gepal/segundosimposio/paulareginape reiramarcelino.pdf (acessado em 02/Mai/2011).

16. Chaves SCL, Santana VS, Leão ICM, Santana JN, Lacerda LMAA. Determinantes da implantação de um programa de segurança e saúde no trabalho. Rev Panam Salud Pública 2009; 25:204-12.

17. Deddeca CS, Rosandiski E. Recuperação econômica e geração de empregos formais. Parcerias Estratégicas 2006; (22):169-90.

18. Instituto Brasileiro de Geografia e Estatística. Pesquisa mensal de emprego - maio 2011. http://www. ibge.gov.br/home/estatistica/indicadores/traba lhoerendimento/pme_nova/pme_201105pubCom pleta.pdf (acessado em 25/Jun/2011).
19. Hennington EA, Cordeiro R, Moreira Filho DC. Trabalho, violência e morte em Campinas, São Paulo, Brasil. Cad Saúde Pública 2004; 20:610-7.

20. Souza MFM, Malta DC, Conceição GMS, Silva MMA, Gazal-Carvalho C, Morais Neto OL. Análise descritiva e de tendência de acidentes de transporte terrestre para políticas sociais no Brasil. Epidemiol Serv Saúde 2007; 16:33-44.

21. Montenegro MMS, Duarte EC, Prado RR, Nascimento AS. Mortalidade de motociclistas em acidentes de trânsito no Distrito Federal do Brasil de 1996-2007. Rev Saúde Pública 2011; 45:529-38.

22. Ahn YS, Bena JF, Bailer AJ. Comparison of unintentional fatal occupational injuries in the Republic of Korea and the United States. Inj Prev 2004; 10:199205.

23. Santamaría N, Catot N, Benavides FG. Time trends in fatal traumatic occupational injuries in Spain (1992-2002). Gac Sanit 2006; 20:280-6.

24. Fabiano B, Currò F, Pastorino R. Occupational injuries in Italy: risk factors and long term trend (195198). Occup Environ Med 2001; 58:330-8.

Recebido em 23/Jan/2013

Versão final reapresentada em 29/Jan/2014

Aprovado em 19/Fev/2014 